\title{
Tratamiento cognitivo-conductual de la adicción a videojuegos de rol online: fundamentos de propuesta de tratamiento y estudio de caso
}

\author{
Clara Marco* y Mariano Chóliz* \\ Facultad de Psicología, Universidad de Valencia
}

\begin{abstract}
Resumen: El propósito de este trabajo es presentar una propuesta de tratamiento cognitivo-conductual para la intervención en la adicción a videojuegos. Se presenta el caso de un varón de 21 años con problemas con el uso de los videojuegos. Se expone el procedimiento en cada una de las fases de las que consta el tratamiento, así como los resultados de su eficacia en un estudio de caso. Después de trece semanas de intervención se aprecian importantes cambios en el nivel de dependencia. Los resultados muestran una disminución significativa del tiempo de uso del ordenador y de juego, así como una mejoría del funcionamiento personal y social del paciente. Palabras clave: Adicción a videojuegos; adicciones tecnológicas; terapia cognitivo-conductal; Massively Multiplayer Online Role Playing Games (MMORPGs).
\end{abstract}

\section{Introducción}

Los videojuegos de rol multijugador masivo online $\mathrm{O}$ MMORPG (Massively Multiplayer Online Role-Playing Game) ofrecen un gran abanico de posibilidades a los jugadores, ya que éstos pueden crear su personaje, interactuar con otros participantes, explorar todo un mundo virtual, llevar a cabo acciones online y, al mismo tiempo, desarrollar las habilidades de su avatar y conseguir subir de nivel en el juego. Se habla de juegos "multijugador" y "masivos" puesto que millones de personas pueden estar jugando al mismo tiempo, y "en línea" porque es necesario estar conectado a Internet para jugar. Los MMORPG son juegos de rol en los que los personajes pueden evolucionar, por ejemplo, en sus atributos y equipamiento. Esta evolución suele plasmarse en un progresivo incremento de "poder" (o progresión de niveles), así como en un constante aprendizaje de nuevas técnicas o aptitudes que permiten al jugador interactuar de forma diferente con su entorno (Llavero, 2010). Algunos ejemplos de estos juegos son $W$ orld of $W$ arcraft (WoW), EverQuest, Ultima Online o Lineage, que cuentan con millones de jugadores en el mundo.

En la sociedad actual los videojuegos constituyen una importante forma de entretenimiento, continúan incrementando su cuota de penetración y su industria lidera el segmento de ocio digital e interactivo en España (aDeSe, 2010), especialmente entre los adolescentes y los jóvenes, quienes suelen dedicar una parte importante de su tiempo a jugar con ellos. Las características específicas de los MMORPG como la percepción de un mundo virtual persistente, la posibilidad de progresar en función del tiempo dedicado al juego, el gran número y variedad de misiones que completar o

* Dirección para correspondencia [Correspondence address]: Dpto. de Psicología Básica. Universidad de Valencia. Avda. Blasco Ibáñez, 21. 46010, Valencia (España).

E-mail: Clara.Marco@uv.es / Mariano.Choliz@uv.es
Title: Cognitive-behavioral treatment of online role-playing videogames: Proposal of treatment and a case study.

Abstract: This article provides a proposal of cognitive-behavioral treatment for video game addiction. A clinical case (a 21 years old man with serious problems caused by the excessive use of videogames) is presented. The intervention phases are explained. The results obtained have been analyzed using a case study design. After thirteen weekly sessions, the patient showed a significant decrease in the amount of time spent using computer and gaming, as well as an improvement of personal and social functioning. The efficacy of the program applied is discussed.

Key words: Videogame addiction; technological addictions; cognitivebehavioral therapy; Massively Multiplayer Online Role Playing Games (MMORPGs).

la organización en grupos o equipos- fomentan el que los jugadores empleen un elevado número de horas en estos juegos y que algunos de ellos lleguen a manifestar serios problemas. Por ejemplo, en el caso de World of $W$ arcraft se estima que la media de horas dedicadas por jugador es de veinticinco por semana, llegándose a más de diez horas diarias (Monnet, 2011) o cincuenta horas semanales, según algunos estudios (Fuster et al., 2012).

A pesar de ello, el concepto de "adicción a videojuegos" genera un considerable debate y posiciones divergentes en cuanto a su consideración como entidad clínica o diagnóstica (Blaszczynski, 2008; Estallo, 2009; Griffiths, 2000, 2008; Tejeiro y Bersabé, 2002; Wood, 2007). Así, mientras que para algunos autores se trata de una forma de adicción comportamental, otros la consideran una faceta del trastorno de control de impulsos o un trastorno comórbido de la depresión o la ansiedad social (Davis, 2001; Ha et al., 2006). A pesar de ello, existe suficiente evidencia empírica, clínica y científica de que el juego excesivo es una actividad potencialmente problemática (Griffiths, 2000; Tejeiro y Bersabé, 2002) y en algunos casos adictiva (Griffiths, 2008). Y esto es así especialmente en los MMORPG, por tratarse de una actividad expansiva y sin fin, en que las posibilidades de acción son innumerables (Chappell, Eatough, Davies y Griffiths, 2006; Thomas y Martin, 2010).

Es muy característico entre los jugadores que llegan a desarrollar un problema de uso excesivo el que dediquen cada vez más tiempo al juego, lo cual va en menoscabo de otras actividades tan importantes como las relaciones interpersonales cara a cara, los estudios o el tiempo dedicado a otras aficiones, con la interferencia que ello puede llegar a generar (Griffiths, 2010). Es frecuente que las relaciones interpersonales queden afectadas, produciéndose discusiones en el núcleo familiar y con la pareja, abandonando actividades de ocio alternativas y descuidando obligaciones como los estudios o el trabajo (Batthyány, Müller, Benker y Wölfling, 2009; Griffiths, Davies y Chappell, 2004; King y Delfabbro 
2009; Liu y Peng, 2009; Peng y Liu, 2010; Phillips, Rolls, Rouse y Griffiths, 1995; Rehbei, Psych, Kleimann, Mediasci y Mossle, 2010; Yee, 2006a, 2006b). Cuando, además, no se puede pasar sin jugar -o cuesta excesivamente dejar de hacerlo-, nos encontramos con un problema de dependencia (Chóliz y Marco, 2012) de una actividad que, en principio era lúdica, pero que se ha convertido en un proceso adictivo similar a la adicción a Internet (Block, 2008) o al móvil (Chóliz, 2010). Es decir, lo que actualmente se denomina adicción tecnológica (Echeburúa, Labrador y Becoña, 2009; Griffiths, 1995).

Otro elemento relevante es que los juegos pueden actuar como distractores de estados de ánimo negativos (DillmanCarpentier, Brown, Bertocci, Forbes y Dahl, 2008) manteniendo así la conducta de juego mediante refuerzo negativo. De hecho, los resultados de varios estudios indican que la adicción a los videojuegos online está relacionada con motivaciones para jugar tales como el sobrellevar emociones negativas, el estrés, el miedo o el escape de situaciones aversivas (Grüsser, Thalemann, Albrech y Thalemann, 2005; Hussain y Griffiths 2009; King y Delfabbro, 2009; Ng y Wiemer-Hastings, 2005; Wan y Chiou, 2006a, 2006b). En resumen, se encuentra una estrecha relación con las motivaciones asociadas con estilos de afrontamiento disfuncionales, con la socialización y la satisfacción personal, que constituyen factores de riesgo para el desarrollo de la adicción a juegos online (Kuss y Griffiths, 2011).

La adicción a los videojuegos -igual que el resto de adicciones tecnológicas- no aparece como categoría diagnóstica en el DSM-IV-TR ni en la CIE-10. No obstante, algunos investigadores consideran que los criterios del trastorno por dependencia de sustancias pueden aplicarse a algunos casos de uso excesivo de videojuegos, constituyendo, por lo tanto, un problema de dependencia aunque no exista una sustancia de por medio (Young, 1998). A pesar de que otros autores optan por asumir los criterios del juego patológico, consideramos que la dificultad en el control de impulsos no es la única característica del abuso y uso excesivo de las "nuevas" tecnologías y que los criterios del trastorno por dependencia se ajustan de forma más apropiada a la sintomatología clínica de estos problemas. De hecho, el juego patológico -que hasta ahora se había incluido en la categoría de los trastornos de control de impulsos del DSM-IV-TR (APA, 2000)- ha pasado a ser clasificado propiamente como trastorno adictivo en la reciente edición del DSM-5 (Petry, 2006; Potenza et al., 2003).

En lo que se refiere a los MMPORG, algunos estudios apuntan la existencia de un pequeño subgrupo de jugadores que cumplen los criterios antes mencionados y que suelen presentar problemas relacionados con el área académica, laboral y las relaciones interpersonales (Chappell et al., 2006; Grüsser, Thalemann y Griffiths, 2007; Lee et al., 2007; Longman, O'Connor y Obst, 2009; Van Rooij, Schoenmakers, Vermulst, Van der Eijden y Van de Mheen, 2010).

En los últimos años ha aumentado el interés y el cuerpo de investigación dedicado a la intervención psicológica en la adicción a videojuegos. En un reciente trabajo, King, Delfabbro, y Griffiths (2009) proponen el uso de autorregistros y el aumento de la conciencia de las consecuencias negativas del uso excesivo del juego, la psicoeducación o la modificación de los pensamientos irracionales como técnicas para el tratamiento de la dependencia de videojuegos, y concluyen que la terapia cognitivo-conductual es una modalidad apropiada para el abordaje de este trastorno (Orzack, Voluse, Wolf y Hennen, 2006; Young, 2007).

Por su parte Young (2009) resalta la importancia de trabajar con la familia del adicto -incluyendo la orientación y psicoeducación de los miembros de la familia, estrategias para hacer frente a la pérdida de confianza, etc.-, especialmente si se trata de un niño o adolescente. Lemmens, Valkenburg y Peter (2011) - encuentran que la incompetencia social, la soledad y la baja autoestima son factores que aumentan la probabilidad de desarrollar la adicción a videojuegos y que, por tanto, la prevención y tratamiento podrían centrarse en actividades que estimulen el desarrollo del bienestar social, la mejora de la autoestima y las relaciones sociales.

Van Rooij (2011) propone la combinación de la terapia cognitivo-comportamental con el uso de la entrevista motivacional y subraya la importancia de trabajar la motivación para el cambio, el establecimiento de un objetivo terapéutico, el aumento del autocontrol, la prevención de recaídas y el entrenamiento en habilidades de afrontamiento.

Estas técnicas de intervención también se han incluido en el tratamiento de la adicción a Internet, para la que existe mayor cantidad de estudios (Chóliz y Marco, 2012; Du, Jiang y Vance, 2010; Kim, 2008; Orzack, et al., 2006; Young, 2007, 2011) que en el caso de la adicción a videojuegos, donde apenas existen trabajos que presenten los detalles de la intervención realizada ni su eficacia, por lo que es necesario continuar investigando en esta línea. En este sentido, los estudios de caso (Allison, von Wahlde, Shockley y Gabbard, 2006; Block, 2007; Griffiths, 2000; Keepers 1990; Kuczmierczyk, Walley y Calhoun, 1987; Wan y Chiou 2006b) pueden ofrecer una primera evidencia sobre la existencia de dicha entidad clínica y sus características, así como promover futuros estudios de $N=1$, que podrían aportar datos que en ocasiones son muy necesarios (Griffiths y Beranuy, 2009).

El presente estudio pretende dar a conocer los resultados de la intervención psicológica en una persona que presenta dependencia de un videojuego multijugador online (MMORPG), a la que se ha aplicado un protocolo de tratamiento cognitivo-conductual.

\section{Método}

\section{Diseño del estudio}

Se empleó un diseño de una sola persona $(N=1)$ del tipo A-B (Barlow y Hersen, 1988), siendo el modo de tratamiento mediante un abordaje en individual con seguimiento. 
Para evaluar los resultados de la intervención se administraron diversos cuestionarios, que el paciente cumplimentó antes y después del tratamiento (medidas pre-post tratamiento y seguimiento). También se analizó la evolución del paciente mediante autorregistros.

\section{Presentación del caso}

\section{Identificación del paciente y motivo de consulta o referencia}

P. es un varón de 21 años, soltero y sin hijos. Procede de una familia de nivel socioeconómico medio, es el pequeño de dos hermanos y reside con sus padres en el domicilio familiar. Su hermano se independizó hace cinco años y la relación entre P. y éste es escasa. La relación con sus padres es problemática, existe poca comunicación entre ellos y las interacciones suelen darse en forma de discusiones la mayoría de las veces. En la actualidad P. estudia un Ciclo Formativo Superior (CFS) -Técnico en Actividades Físicas y Animación Deportiva-.

Respecto al motivo de consulta, los padres de P. contactan con la Unidad de Investigación de Adicción al Juego y Adicciones Tecnológicas tras mantener una conversación con el tutor de su hijo y saber que éste no está asistiendo a clase ni está haciendo los trabajos que se le solicitan. P. acude presionado por su familia que refiere que "es adicto a un videojuego en red'.

En la primera sesión, el paciente firmó el consentimiento informado y se le explicó el compromiso del terapeuta con el articulado del Código Deontológico, haciendo especial hincapié en la confidencialidad y el secreto profesional.

\section{Estrategias de evaluación}

Se realizó una evaluación multimétodos, se llevaron a cabo tres entrevistas clínicas semiestructuradas, la primera de ellas con el padre y la madre de P. y las otras dos con el joven. Se registró el número de horas que pasaba en el ordenador y jugando, y se evaluó la interferencia producida en los estudios y en el cumplimiento de sus responsabilidades académicas a través de los resultados obtenidos en los exámenes realizados, la asistencia a clase y a las prácticas -para ello se mantuvo contacto con el tutor académico de P., que sirvió como criterio externo de evaluación-.

Se administró el Test de Dependencia de Videojuegos (TDV) (Chóliz y Marco, 2011), un instrumento diagnóstico de la dependencia de videojuegos basado en los principales criterios diagnósticos del trastorno por dependencia de sustancias del DSM-IV-TR (APA, 2000). El cuestionario está formado por 21 ítems y consta de cuatro factores: Abstinencia, Abuso y Tolerancia, Problemas Ocasionados por los Videojuegos, y Dificultad en el Control. El alfa de Cronbach del conjunto de ítems que forman el cuestionario es de 0.94 , por lo que podemos considerar que se trata de un instrumento con características psicométricas satisfactorias.
Al finalizar el tratamiento se administró el Inventario de Alianza Terapéutica y Teoría del Cambio, WATOCI (Duncan y Miller, 1999), en la versión española de Corbella y Botella (2004). Desde cualquier enfoque psicoterapéutico y, especialmente en el tratamiento de las adicciones y la Entrevista Motivacional, la relación terapeuta-paciente y el establecimiento de una buena alianza entre ambos resulta fundamental. Este inventario consta de 17 ítems y está formado por 4 subescalas: Acuerdo en las tareas, Acuerdo en los objetivos, Vinculo positivo y Teoría del cambio. El alfa de Cronbach del conjunto de ítems que forman el cuestionario es de 0.93 (Corbella y Botella, 2004).

Se llevó a cabo un Análisis Funcional de la Conducta (O'Neill, Horner, Albin, Storey y Sprague, 1990), a través de un proceso de auto-observación -además de la información obtenida de diversas fuentes en las entrevistas-, para lo que se utilizó un procedimiento de autorregistro como el que se muestra en la Tabla 1, con columnas referidas a la fecha y situación, nombre del juego o aplicación de Internet usada, tiempo dedicado, emoción, pensamientos, conducta y consecuencias, que son los principales parámetros implicados en el Análisis Funcional. La duración de las sesiones de uso del ordenador y videojuegos se midió con un reloj y la frecuencia se operativizó teniendo en cuenta el número de veces que se conectaba al día. Cada vez que P. dejaba de jugar y retomaba el juego más tarde se consideró como una sesión de juego diferente y debía anotarla en la hoja de autorregistro. Se pidió que toda esta información fuera transcrita a diario, inmediatamente después de cada sesión de juego y que por la noche la repasara para contar con la posibilidad de completar o corregir algún dato.

Tabla 1. Autorregistro del uso del ordenador.

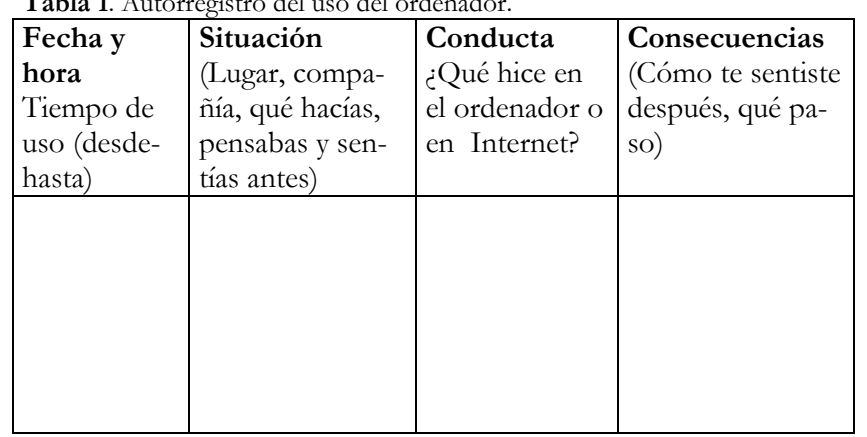

\section{Formulación clínica del caso}

\section{Historia y evolución del problema}

P. refiere que comenzó a jugar a un juego de rol online (Lineage II, un juego MMORPG) hace unos dos años, cuando un amigo se lo enseñó. Empezó a jugar para divertirse, entretenerse y combatir el aburrimiento, y a usar las redes sociales para estar en contacto con amigos y conocidos. Respecto al juego, a medida que iba avanzando aumentó su "autoexigencia" y pensaba que "quería ser el mejor". Al cabo de 
unos meses la frecuencia de juego se había incrementado hasta diez horas diarias, aproximadamente.

En las entrevistas iniciales fueron apareciendo síntomas que hacían sospechar de un problema de dependencia, según los criterios del DSM-IV-TR. Así, por ejemplo, el tiempo dedicado fue incrementándose llegando a jugar unas entre diez y doce horas diarias. También indicaba que a veces no era capaz de evitar jugar aunque quisiera; además refería que mientras realizaba otras actividades continuaba pensando en el juego, que nunca se puso un límite de tiempo o un horario y que, con el paso del tiempo, la frecuencia y duración de la conducta de juego fue en aumento. P. afirma que cuando no puede jugar se siente muy aburrido y "le apetece mucho jugar", y que cuando más ha jugado ha sido en vacaciones o periodos en que ha tenido más tiempo libre.

Respecto a las consecuencias del uso excesivo, P. reconoce haber dejado de quedar con sus amigos algunas veces por estar jugando; haber dejado de ir a clase en numerosas ocasiones; no haber estudiado y no haber presentado los trabajos de clase. Además, el juego es con frecuencia un motivo de discusión con sus padres.

P. refiere usar el ordenador (para conectarse a Internet) alrededor de diez horas al día, aunque su madre afirma que lo habitual es que permanezca más tiempo (alrededor de catorce horas).

\section{Diagnóstico}

Se realiza un diagnóstico de Dependencia de Internet y Videojuegos, cumpliendo en el momento de la evaluación los siguientes criterios:

- Abstinencia: experimenta malestar cuando lleva un tiempo sin jugar o existen impedimentos para ello.

- Usa Internet y el juego más tiempo del que tenía previsto

- No puede dejar de usar Internet ni "cortar" con el juego

- Emplea excesivo tiempo en todo lo relacionado con Internet y el videojuego
- Ha dejado de hacer cosas por dedicar tanto tiempo a Internet y al juego (ha dejado de ir a clase, ha suspendido la primera evaluación del módulo que está estudiando, etc.).

- Sigue conectándose a pesar de saber que le está perjudicando.

De acuerdo con los postulados básicos del Análisis Funcional de Conducta (O'Neill et al., 1990) centramos la atención en la conducta observable y mensurable (conectarse a Internet para jugar y tiempo de permanencia en el juego), así como las consecuencias de dicha actividad, como el alivio del aburrimiento y de las preocupaciones. Igualmente, se registra cuando la conducta le sirve para "desconectar" de las discusiones que existen en su núcleo familiar, entendiendo que en gran medida el problema se mantiene por un proceso de reforzamiento negativo.

\section{Tratamiento}

El tratamiento aplicado es de corte cognitivo-conductual. La intervención terapéutica fue individual y se realizó durante trece sesiones, con una periodicidad de una vez por semana -las sesiones tuvieron una duración aproximada de una hora-. Siguiendo la estructura del tratamiento propuesto por Chóliz y Marco (2012) para la adicción a Internet y redes sociales, el tratamiento se estructuró en varias fases (Tabla 2): I. Fase motivacional y de valoración psicosocial; II. Fase de superación del deseo y síndrome de abstinencia; III. Cambios conductuales y actitudinales; IV. Consolidación y prevención de recaídas; V. Seguimiento. En todas las sesiones se reservó un tiempo para contrastar información con la familia de P. y para ofrecerles pautas de intervención. Además, se mantuvo contacto periódicamente con el tutor de P. para contar con otra fuente de información.

Se realizaron seguimientos al mes y a los tres meses de producirse el alta terapéutica.

Tabla 2. Fases, sesiones, contenidos, técnicas y tareas para casa.

\begin{tabular}{|c|c|c|}
\hline Fase & Sesión & Plan de las sesiones \\
\hline $\mathrm{I}$ & 1 & Entrevista inicial. Evaluación con la familia \\
\hline $\mathrm{I}$ & 2 & Autorregistro uso de Internet/juego \\
\hline$\overline{\mathrm{I}}$ & 3 & $\begin{array}{l}\text { Finalización de la evaluación. Detalles formales de laAutorregistro uso Internet/juego, listado de posibles } \\
\text { terapia } \\
\text { actividades de ocio y cambios a realizar en el núcleo } \\
\text { familiar, y planificación (horario) de los trabajos y es- } \\
\text { tudio }\end{array}$ \\
\hline I y II & 4 & 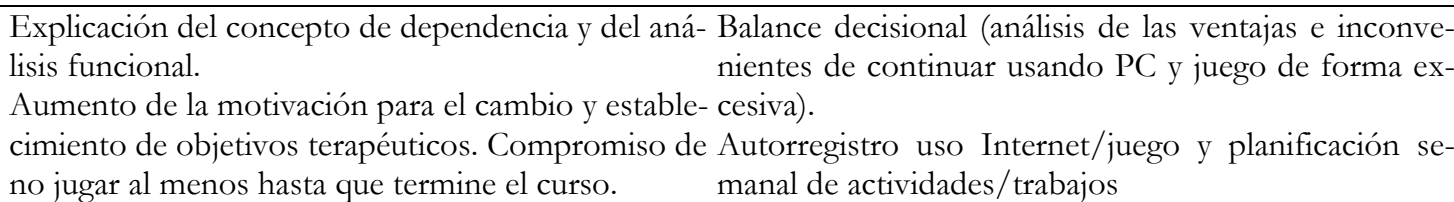 \\
\hline$\overline{\mathrm{II}}$ & 5 & $\begin{array}{l}\text { Contrato conductual para alcanzar los objetivos: re- Autorregistro, aplicar las pautas del contrato, planifi- } \\
\text { ducir horas de uso del ordenador, aprobar el curso y cación trabajos y estudio. Se establecen normas en ca- } \\
\text { mejorar la convivencia. } \\
\text { sa respecto a la relación padres-hijo: se acuerda comer } \\
\text { juntos en la mesa, hablar de temas que no sean con- } \\
\text { flictivos, hablarse de forma menos "violenta", hacien- }\end{array}$ \\
\hline
\end{tabular}




\begin{tabular}{|c|c|c|c|}
\hline & & & do peticiones concretas. \\
\hline II & 6 & $\begin{array}{l}\text { Control de estímulos (horario de uso del ordenador, } \\
\text { uso exclusivo para realizar trabajos, hora de apagado } \\
\text { por la noche, supervisión de los padres). } \\
\text { Supervisión del cumplimiento del contrato }\end{array}$ & $\begin{array}{l}\text { Autorregistro, aplicación del contrato, planificación } \\
\text { trabajos y estudio. } \\
\text { Compromiso del paciente de asistir a clase y entregar } \\
\text { los trabajos pendientes. }\end{array}$ \\
\hline II & 7 & $\begin{array}{l}\text { Control de estímulo. } \\
\text { Supervisión del cumplimiento del contrato Estrate- } \\
\text { gias de afrontamiento }\end{array}$ & $\begin{array}{l}\text { Autorregistro, aplicación del contrato, planificación } \\
\text { trabajos y estudio. Pensar actividades de ocio y tiempo } \\
\text { libre, y "normas" de casa (P. fregará sus platos y bajará } \\
\text { su ropa sucia, a cambio de un menor control por parte } \\
\text { de su madre). }\end{array}$ \\
\hline II y III & 8 & $\begin{array}{l}\text { Supervisión y nuevas pautas para reducir tiempo } \\
\text { dedicado al ordenador. } \\
\text { Establecimiento de "normas" para la convivencia } \\
\text { / contrato }\end{array}$ & $\begin{array}{l}\text { Autorregistro, aplicación del contrato, planificación } \\
\text { trabajos y estudio } \\
\text { Se añaden las siguientes pautas: si va a estar más tiem- } \\
\text { po del previsto inicialmente en el PC tendrá que avi- } \\
\text { sar, irá a la piscina aunque tenga que hacer otras cosas } \\
\text { y se intentará reducir el tiempo de uso del PC una ho- } \\
\text { ra. }\end{array}$ \\
\hline II y III & 9 & $\begin{array}{l}\text { Supervisión y nuevas pautas (ha vuelto a jugar). Va- } \\
\text { loración de los cambios realizados y cambios pen- } \\
\text { dientes o a realizar. }\end{array}$ & $\begin{array}{l}\text { Autorregistro, aplicación del contrato, planificación } \\
\text { actividades. } \\
\text { Se planifica qué va a hacer cuando se conecte a Inter- } \\
\text { net, se establece un tiempo máximo diario para el uso } \\
\text { del PC y se usa la demora forzada. }\end{array}$ \\
\hline III y IV & 10 & $\begin{array}{l}\text { Supervisión y refuerzo de la reducción del número } \\
\text { de horas de juego/ordenador. Acuerdo de reduc- } \\
\text { ción de horas ordenador. } \\
\text { Valoración cambios realizados e introducción PR }\end{array}$ & $\begin{array}{l}\text { Autorregistro } \\
\text { Tarea para identificar situaciones de alto riesgo } \\
\text { Planificación de actividades alternativas de ocio. }\end{array}$ \\
\hline III y IV & 11 & $\begin{array}{l}\text { Supervisión y abordaje de la situación de conflicto } \\
\text { familiar. Compromiso de jugar menos horas } \\
\text { PR }\end{array}$ & $\begin{array}{l}\text { Autorregistro } \\
\text { Cumplimiento del contrato } \\
\text { Demora forzada: tiene que esperar } 5 \text { minutos al llegar } \\
\text { a casa antes de ponerse a usar el ordenador. } \\
\text { Análisis de situaciones de alto riesgo }\end{array}$ \\
\hline IV & 12 & $\begin{array}{l}\text { Supervisión y prevención de recaídas } \\
\text { Propuesta de "normas mínimas" para la convivencia } \\
\text { PR }\end{array}$ & $\begin{array}{l}\text { Autorregistro } \\
\text { En sesión se plantea a P. y sus padres que piensen si } \\
\text { quieren seguir o no en terapia, ya que no discuten con- } \\
\text { tinuamente en las sesiones. } \\
\text { Análisis de las ventajas de abandonar el juego. } \\
\text { Efecto de violación de la abstinencia. }\end{array}$ \\
\hline IV & 13 & Supervisión y prevención de recaídas & Citación para seguimiento \\
\hline$\overline{\mathrm{V}}$ & 14 & Seguimiento 1 (al mes) & Citación para segundo seguimiento \\
\hline $\mathrm{V}$ & 15 & $\begin{array}{l}\text { Seguimiento (vía correo electrónico, a los tres me- } \\
\text { ses). }\end{array}$ & \\
\hline
\end{tabular}

Factores relacionados con el terapeuta

Las sesiones fueron conducidas por una única terapeuta, autora del presente trabajo y con formación específica en adicciones comportamentales.

La relación terapéutica podría considerarse buena. El paciente mostró adhesión al tratamiento y trató de cumplir con las prescripciones. No obstante, al inicio de la intervención no siempre completó los registros solicitados. El paciente no canceló las citas programadas, y siempre avisó si se veía obligado a cambiarlas.

\section{Resultados}

\section{Fase de Tratamiento}

Parámetros objetivos: frecuencia de uso y resultados de cuestionarios

A continuación se presentan los resultados del cuestionario TDV así como la evolución del tiempo de uso del ordenador y juego (Figuras 1 y 2, y Tablas 3 y 4). 


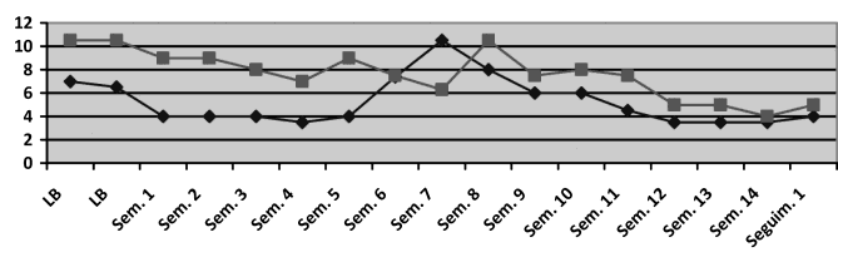

$\rightarrow-$ Horas PC entre semana $\rightarrow-$ Horas PC fin seman

Figura 1. Evolución del tiempo de uso del ordenador entre semana y en fin de semana.

En la Figura 1 se aprecia que durante las cinco primeras semanas se produce una disminución del tiempo que P. usa el ordenador, tanto entre semana como en fin de semana. Esta disminución coincide con el acuerdo alcanzado en terapia de mantenerse sin jugar hasta que termine el periodo de exámenes, con la reducción del tiempo de uso del ordenador y el establecimiento de una hora concreta para apagarlo durante la noche. Se mantiene el patrón de mayor uso durante el fin de semana.

Durante la sexta semana se produce un aumento de las horas que pasa en el ordenador entre semana, el paciente refiere que se debe a que ha estado haciendo trabajos con el mismo.

En la séptima y octava semana aumenta el tiempo de uso del ordenador entre semana y en fin de semana, respectivamente. Este aumento coincide con el momento en que P. termina los exámenes (ha recuperado todos los exámenes y ha superado dos pruebas de natación) y vuelve a jugar a $L i$ neage II.

A partir de ese momento, se produce una reducción de las horas de uso de ordenador y juego, que se mantiene estable hasta el final de la intervención. En la decimotercera sesión, P. refiere haber abandonado de nuevo el juego y se mantiene sin jugar hasta el seguimiento.

En la Tabla 3 y Figura 2 aparece la puntuación obtenida por el paciente en el TDV en el pretest y después del tratamiento. La evolución de los resultados indica una mejoría en cuanto a la dependencia de videojuegos, ya que, mientras que en la evaluación inicial la puntuación directa de P. en el TDV es de 81, en el retest la puntuación obtenida es 44. En este último momento, P. ya no cumple los criterios diagnósticos de dependencia de videojuegos. Las mejorías más significativas se producen en los Factores I - Abstinencia- y II Abuso y Tolerancia-, es decir, el paciente ya no experimenta tanto malestar cuando no puede jugar con videojuegos y juega menos que antes.

Tabla 3. Puntuaciones pre y post-tratamiento en el cuestionario TDV.

\begin{tabular}{l|ccc}
\hline \multicolumn{2}{l}{ Cuestionario } & Pretest & Postest \\
\hline TDV Total $(0-100)$ & 81 & 44 \\
Factor I $\quad(0-40)$ & 32 & 18 \\
Factor II $\quad(0-20)$ & 19 & 2 \\
Factor III $\quad(0-16)$ & 12 & 11 \\
Factor IV & $(0-24)$ & 18 & 13 \\
\hline
\end{tabular}

*Los números entre paréntesis corresponden a los rangos de las puntuaciones.

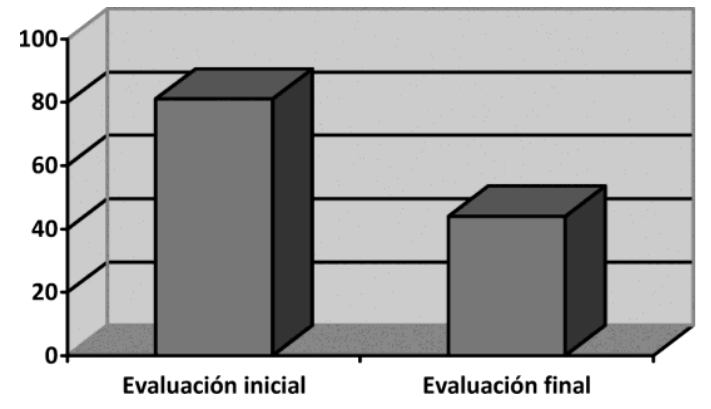

口TDV

Figura 2. Puntuaciones pre y post-tratamiento en el cuestionario TDV.

Por otra parte, los resultados del inventario WATOCI (Tabla 4) apuntan la existencia de una buena relación terapéutica puesto que existe un alto acuerdo entre la terapeuta y el paciente tanto en los objetivos como en las tareas de la terapia.

Tabla 4. Resultados del inventario WATOCI para la evaluación de la alianza terapéutica.

\begin{tabular}{lcc}
\hline Factor & $\begin{array}{c}\text { Puntuación } \\
\text { directa }\end{array}$ & $\begin{array}{c}\text { Puntuación mínima y máxima } \\
\text { en la escala/cuestionario }\end{array}$ \\
\hline Tareas & 24 & 4 a 28 \\
Vínculo Positivo & 28 & 4 a 28 \\
Objetivos & 26 & 4 a 28 \\
T ${ }^{\text {a cambio }}$ & 31 & 5 a 35 \\
(Total) & 109 & 17 a 119 \\
\hline
\end{tabular}

\section{Análisis cualitativo}

Cuando P. acude a la primera entrevista de evaluación refiere haber dejado de jugar a Lineage II recientemente y "baberse dado cuenta de que estaba viciado". Tras la valoración psicosocial P. se compromete a no volver a jugar, al menos hasta que haya acabado el curso, para así poder centrarse en asistir a clase, realizar los trabajos que tiene pendientes y estudiar para los exámenes.

En la tercera sesión, al preguntarle por el juego, P. refiere que, "aunque no es fácil" porque "nota que le falta algo", sigue sin jugar. Es capaz de reflexionar y concluir que "cuando se pone a jugar se engancha y pierde mucho tiempo". En la cuarta sesión sigue afirmando que se mantiene sin jugar "pero que piensa en algunos momentos en el juego, y que cree que acabará volviendo a jugar" ya que "nota la ausencia del juego". Refiere que "si volviera a jugar, trataría de controlar la situación y aprender de lo ocurrido, aunque no sabe si podría". Al final de dicha sesión, su padre valora que P. ha mejorado y que está usando menos el ordenador. Se produce un aumento de la conciencia y aceptación del problema.

Desde el principio de la intervención y hasta la octava sesión, P. se mantiene sin jugar y el tiempo de uso del ordenador se reduce -la disminución es más significativa entre semana que en fin de semana- (Figura 1).

En la octava sesión P. dice que ha vuelto a jugar a Lineage II. Refiere que "ahora no lo ve tan interesante y que se aburre", 
además valora que "antes estaba mucho más viciado". En la novena sesión afirma seguir jugando, "pero no es como antes, mientras estoy en el ordenador hago más cosas...no solamente juego". Refiere "mi prioridad ahora son las prácticas". En la décima sesión, P. explica que su forma de jugar ha cambiado: "el nivel de enganche no es el mismo de antes... además, ahora me aburro y tengo que ir a las prácticas". Valora que el juego no interfiere con el cumplimiento de sus responsabilidades y obligaciones en este momento. En la undécima sesión P. reconoce que está jugando "pero menos que antes".

En la duodécima sesión, P. refiere haber reducido las horas de juego durante la última semana. Sigue afirmando que "ahora no está como antes con el juego", "ahora es una forma de hablar con amigos...". Además, plantea que "probablemente deje de jugar", "porque para avanzar necesitaría más horas, me veo retrasado...y me aburre un poco".

El tiempo de uso del ordenador aumenta cuando retoma el juego y, después, se vuelve a producir una reducción progresiva del tiempo invertido en el juego e Internet, conforme lo acordado en la terapia.

En la decimotercera sesión P. cuenta que ha dejado de jugar, "no tengo tiempo... y no creo que vuelva a jugar".

\section{Normalización de otros aspectos de su vida}

En el área académica cabe destacar que cuando P. acude por primera vez la situación es complicada: no está acudiendo a clase, no entrega los trabajos pendientes y ha suspendido tres asignaturas de la primera evaluación. Con el tratamiento recupera las asignaturas pendientes y aprueba todas las de la segunda evaluación. También supera las pruebas de natación que no había logrado aprobar en la primera convocatoria. Posteriormente, realiza durante varios meses las prácticas en un centro deportivo, mostrándose responsable y cumpliendo con los horarios y actividades que tiene asignados.

Se contrasta la mejoría del paciente con su tutor, que valora que " $P$. ha realizado un gran cambio: se muestra más abierto con sus compañeros, su actitud es más positiva, y ha salvado el curso. Lo encuentro mucho mejor".

En el área social, se logra que P. se interese más por quedar y salir con sus amigos. $\mathrm{Y}$ aunque las relaciones familiares continúan siendo complicadas al final de la intervención, se logra cierta mejoría y la asunción por parte de todos los miembros de la familia de unas normas mínimas para la mejora de la convivencia.

Teniendo en cuenta toda esta información, se puede afirmar que la evolución del paciente es positiva. En el Anexo I puede consultarse la entrevista realizada al paciente para evaluar el programa de tratamiento.

\section{Fase de seguimiento}

Tras haber finalizado las prácticas que estaba haciendo, P. obtiene el título de Técnico en Actividades Físicas y Animación Deportiva. Se plantea descansar en verano y en sep- tiembre elaborar su curriculum, buscar trabajo o seguir formándose.

Continúa sin jugar y refiere estar saliendo más con sus amigos. Respecto al ordenador, piensa que en verano quizá aumente su uso, ya que tendrá más tiempo libre. Se le recuerdan algunas de las principales pautas para el uso adecuado del ordenador e Internet.

En el momento del primer y segundo seguimiento refiere utilizar el ordenador algo más de cuatro horas al día.

\section{Conclusiones y discusión}

Este trabajo se basa en la asunción de que los videojuegos, especialmente los MMPORG, tienen una serie de características que pueden llegar a provocar problemas de dependencia en algunas personas que se implican demasiado en ellos o los utilizan excesivamente. Describimos un protocolo de tratamiento cognitivo-conductual que ha sido implementado en un caso clínico.

Se asume que el caso que se presenta se trata de un problema adictivo puesto que, teniendo en cuenta los criterios del trastorno por dependencia de sustancias, el paciente presenta ante los videojuegos las principales manifestaciones que se asumen para el diagnóstico de un trastorno por dependencia tales como: 1) Tolerancia: cada vez necesita jugar durante más tiempo o a más juegos, de tal forma que el patrón de uso inicial deja de ser suficiente; 2) Abstinencia: malestar significativo cuando interrumpe el juego o cuando se lleva un tiempo sin jugar; 3) Jugar más de lo que se pretendía inicialmente; 4) No poder dejar de jugar, a pesar de que lo intenta; 5) Emplear excesivo tiempo en todo lo relacionado con los videojuegos, hasta tal punto que se llega a producir una interferencia con actividades cotidianas; 6) Dejar de hacer otras actividades o perder oportunidades por jugar; y 7) Seguir jugando a pesar de saber que está siendo perjudicial.

En congruencia con la literatura científica y con investigaciones en las que se demuestra la eficacia de los tratamientos cognitivo-conductuales para la intervención en adicciones comportamentales, se desarrolló un tratamiento basado en dichos principios motivacionales y del aprendizaje para un caso clínico. Tanto la estructura del protocolo de intervención, como las técnicas utilizadas a lo largo de la misma, son una adaptación del modelo de tratamiento cognitivoconductual utilizado para el tratamiento de la adicción a Internet (Chóliz y Marco, 2012). Pueden distinguirse cinco procesos en el tratamiento: a) evaluación y análisis del problema; b) control de estímulo y superación del deseo; c) entrenamiento en técnicas de control y en utilización apropiada de videojuegos; d) fomento de conductas alternativas al uso de videojuegos y e) entrenamiento en prevención de recaídas.

En el proceso de evaluación se utilizaron cuestionarios específicos de dependencia validados en población española, entrevistas semiestructuradas atendiendo a los criterios del DSM-IV-TR para este tipo de problemas, así como el Análisis Funcional de la Conducta. De los resultados obtenidos en 
los principales criterios (horas de uso y días de la semana que juega), así como del análisis cualitativo de algunas de las cuestiones más significativas del proceso de tratamiento, se presenta una propuesta de intervención para el tratamiento de la adicción a videojuegos online, ya que el paciente obtuvo mejoría en los principales indicadores y sintomatología clínica, tales como:

1. Se produce un aumento de la toma de conciencia del problema existente y de sus consecuencias, tal y como relata el paciente en las entrevistas realizadas durante todo el proceso terapéutico.

2. A lo largo de la intervención tiene lugar un descenso del tiempo dedicado al ordenador y al juego, logrando el abandono de éste último en distintos periodos. Hay que tener en cuenta que lo que se pretende en la intervención en las adicciones tecnológicas no es la abstinencia, sino su uso controlado.

3. Se observa una mejoría muy relevante en áreas significativas de su vida personal y académica que estaban viéndose afectadas por el uso excesivo del ordenador y el juego, como los estudios, las relaciones interpersonales y familiares.

4. El cambio objetivo logrado puede observarse comparando la puntuación obtenida por el paciente en el TDV en el pretest y después de la intervención terapéutica postest-. Dicho cambio indica una mejoría en cuanto a la dependencia de videojuegos, ya que mientras que en la evaluación inicial la puntuación directa de P. en el TDV es de 81 , en el restest la puntuación obtenida es 44 y este último momento el paciente ya no cumple los criterios de dependencia de videojuegos.

5. El cambio subjetivo percibido por el propio paciente también es personalmente significativo, ya que éste valora que tiene un mayor autocontrol y que "el nivel de enganche (con el juego) no es el mismo de antes”. El paciente es capaz de priorizar actividades y cumplir sus obligaciones.

En resumen, el caso presentado ilustra algunas de las consecuencias negativas que puede conllevar la dependencia de videojuegos (Griffiths, Davies y Chappell, 2004; King y Delfabbro 2009), y que está llevando a los psicólogos a preocuparse y ocuparse de esta problemática. Existen ya algunas unidades especializadas en la investigación y trata-

\section{Referencias}

Allison, S. E., von Wahlde, L., Shockley, T., y Gabbard, G. O. (2006). The development of the self in the era of the Internet and role-playing fantasy games. American Journal of Psychiatry, 163, 381-385.

American Psychiatric Association (2000). Diagnostic and Statistical Manual of Mental Disorders (4 $4^{\text {th }}$ ed., text revision). Washington, DC: American Psychiatric Association

Asociación de Distribuidores y Editores de Software de Entretenimiento (2010). Anuario $2010 . \quad$ Recuperado http://www.adese.es/pdf/anuario2010/ANUARIO2010.pdf

Barlow, D. H., y Hersen, M. (1988). Diseños experimentales de caso único. Barcelona: Martínez Roca miento de las adicciones tecnológicas, aunque no cabe duda que es necesario continuar investigando en este campo para lograr alcanzar datos consistentes referentes al tratamiento de elección de la adicción a videojuegos, puesto que la mayoría de los estudios que analizan esta problemática coinciden en la necesidad de un tratamiento psicológico, pero los estudios publicados todavía no presentan detalles de las intervenciones aplicadas ni tampoco de su eficacia. Este trabajo presenta una propuesta de tratamiento que puede ser de utilidad para la adicción a videojuegos (más concretamente, MMPORG) basado en técnicas de modificación de conducta, acompañadas de entrevista motivacional.

Consideramos que este trabajo contribuye al desarrollo del necesario cuerpo de conocimientos relativo al tratamiento psicológico de las adicciones tecnológicas, ya que los estudios de caso único permiten referir con gran detalle casos particulares y representan una significativa contribución científica, con gran influencia en el desarrollo, difusión y evaluación de nuevas formas de intervención (Virués-Ortega y Moreno-Rodríguez, 2008).

El presente trabajo tiene una serie de limitaciones por las características del diseño de caso único. Es evidente que los resultados y conclusiones deben ser tomados con precaución y contrastados en estudios con un mayor control experimental, ya que no fue viable controlar la influencia de variables extrañas con capacidad de afectar a los efectos del tratamiento. Otro de los problemas es la falta de un observador o evaluador independiente. Resulta imprescindible seguir investigando en el tratamiento de este trastorno, y de forma ideal, la investigación futura de estudios de casos y de casos clínicos debe tratar de incorporar otros diseños más complejos como por ejemplo, el de línea base múltiple- que permitan una mayor seguridad en la interpretación de los cambios producidos por la terapia psicológica. A pesar de ello, los resultados encontrados parecen sostener la utilidad de las técnicas cognitivo-conductuales en la intervención psicológica de la adicción a videojuegos.

Agradecimientos.- Este trabajo ha sido posible gracias al proyecto de investigación "Desarrollo de un protocolo de tratamiento para la adicción a Internet: Redes sociales y videojuegos online", financiado por la Fundación Universitaria Oriol-Urquijo.

Batthyány, D., Müller, K. W., Benker, F., y Wölfling, K. (2009). Computer game playing: clinical characteristics of dependence and abuse among adolescents. Wiener Klinsche Wochenschrift, 121, 502-509.

Blaszczynski, A. (2008). Commentary: A response to "Problems with the concept of video game "addiction": Some case study examples". International Journal of Mental Health and Addiction, 6, 179-181.

Block, J. J. (2007). Pathological computer game use. Psychiatric Times, 24 (3). Recuperado de http://www.psychiatrictimes.com/pathologicalgambling/content/article/10168/55406?pageNumber $=2$

Block, J. J. (2008). Issues for DSM-V: Internet addiction. American Journal of Psychiatry, 165, 306-307. 
Chappell D., Eatough V., Davies M. N. O., y Griffiths M. D. (2006). EverQuest-it's just a computer game right? An interpretative phenomenological analysis of online gaming addiction. International Journal of Mental Health and Addiction, 4, 205-216.

Chóliz, M. (2010). Mobile phone addiction: a point of issue. Addiction, 105, 373-374.

Chóliz, M. y Marco, C. (2011). Patrón de Uso y Dependencia de Videojuegos en Infancia y Adolescencia. Anales de Psicología, 27, 418-426

Chóliz, M. y Marco, C. (2012). Adicción a Internet y Redes sociales. Tratamiento psicológico. Madrid: Alianza Editorial.

Corbella, S. y Botella, L. (2004). Psychometric properties of the Spanish version of the Working Alliance Theory of Change Inventory (WATOCI). Psicothema, 16, 702-705

Davis, R. A. (2001). A cognitive-behavioral model of pathological Internet use. Computers in Human Behavior, 17, 187-195.

Dillman-Carpentier, F., Brown, J., Bertocci, M., Silk, J., Forbes, E., y Dahl, R. (2008). Sad kids, sad media? Applying mood management theory to depressed adolescents' use of media. Media Psychology, 11, 143-166.

Du, Y., Jiang, W., y Vance, A. (2010). Longer term effect of randomized, controlled group cognitive behavioral therapy for Internet addiction in adolescent students in Shanghai. The Australian and New Zealand Journal of Psychiatry, 44, 129-134.

Duncan, B.L. y Miller, S.D. (1999). Working Alliance Theory of Change Inventory (WATOCI). Recuperado de http://www.talkingcure.com/

Echeburúa, E., Labrador, F., y Becoña, E. (2009). Adicción a las nuevas tecnologias en adolescentes y jóvenes. Madrid: Pirámide.

Estallo, J. A. (2009). El diagnóstico de "adicción a videojuegos": uso, abuso y dependencia. En E. Echeburúa, F., Labrador y E. Becoña (Eds.), Adicción a las nuevas tecnologías en adolescentes y jóvenes (pp.151-166). Madrid: Pirámide.

Fuster, H., Oberst, U., Griffiths, M., Carbonell, X., Chamarro, A., y Talam, A. (2012). Psychological motivation in online role-playing games: A study of Spanish World of Warcraft players. Anales de Psicologia, 28, 274280

Griffiths, M. D. (1995). Technological addictions. Clinical Psychology Forum, 76, 14-19.

Griffiths, M. D. (2000). Does internet and computer "addiction" exist? Some case study evidence. CyberPsychology \& Behavior, 3, 211-218.

Griffiths, M. D. (2008). Videogame Addiction: Further Thoughts and Observations. International Journal of Mental Health and Addiction, 6, 182-185.

Griffiths, M. D. (2010). The role of context in online excess and addiction: Some case study evidence. International Journal of Mental Health Addiction, $8,119-125$.

Griffiths, M. D. y Beranuy, M. (2009). “Adicción a los videojuegos: una breve revisión psicológica". Revista de Psicoterapia, 73, 33-49.

Griffiths, M. D., Davies, M. N. O., y Chappell, D. (2004). Demographic factors and playing variables in online computer gaming. Cyberpsychology \& Behavior, 7, 479-487.

Grüsser S. M., Thalemann R., y Griffiths M. D. (2007). Excessive computer game playing: evidence for addiction and aggression? Cyberpsychology \& Behavior, 10, 290-302.

Grüsser, S. M., Thalemann, R., Albrecht, U., y Thalemann, C. N. (2005). Exzessive Computernutzung im Kindesalter-Ergebnisse einer psychometrischen Erhebung. Wiener Klinische Wochenschrift, 117, 188-195.

Ha, J. H., Yoo, H. J., Cho, H., Chin, B., Shin, D., y Kim, J. H. (2006). Psychiatric comorbidity assessed in Korean children and adolescents who screen positive for Internet addiction. Journal of Clinical Psychiatry, 67, $821-826$.

Hussain, Z. y Griffiths, M. D. (2009). Excessive use of massively-multiplayer online role-playing games: a pilot study. International Journal of Mental Health and Addiction, 7, 563-571.

Keepers, G. A. (1990). Pathologicical preoccupation with video games. Journal of the American Academy of Child and Adolescent Psychiatry, 29, 49-50.

Kim, J. (2008). The effect of a R/T group counselling program on the Internet addiction level and self-esteem of Internet addiction university students. International Journal of Reality Therapy, 17, 4-12.

King, D. L. y Delfabbro, P. (2009). Motivational differences in problem video game play. Journal of CyberTherapy \& Rehabilitation, 2, 139-149.
King, D., Delfabbro, P., y Griffiths, M. D. (2009). The psychological study of video game players: Methodological challenges and practical advice. International Journal of Mental Health and Addiction, 7, 555-562.

Kuczmierczyk, A. R., Walley, P. B., y Calhoun, K. S. (1987). Relaxation training, in vivo exposure and response-prevention in the treatment of compulsive video-game playing. Scandinavian Journal of Behavior Therapy, 16, 185-190.

Kuss, D. y Griffiths, M. D. (2011). Internet gaming addiction: A systematic review of empirical research. International Journal of Health and Addiction 10, $1-19$

Lee, M., Ko, Y. H., Song, H. S., Kwon, K. H., Lee, H. S., Nam M., y Jung, I. K. (2007). Characteristics of internet use in relation to game genre in Korean adolescents. Cyberpsychology \& Behavior, 10, 278-285.

Lemmens, J. S., Valkenburg, P. M., y Peter, J. (2011). Psychosocial causes and consequences of pathological gaming. Computers in Human Behavior 27, 144-152.

Liu, M. y Peng, W. (2009). Cognitive and psychological predictors of the negative outcomes associated with playing MMOGs (Massively Multiplayer Online Games). Computers in Human Behavior, 25, 1306- 1311.

Llavero, I. (2010). MMORPG, impacto económico y social de los mundos virtuales. Universitat Politècnica de València. Escuela Técnica Superior de Ingeniería Informática. Recuperado de http://hdl.handle.net/10251/8626

Longman H., O'Connor, E., y Obst, P. (2009). The effect of social support derived from world of warcraft on negative psychological symptoms. Cyberpsychology \& Behavior, 12, 563-566.

Monnet, V. (2011). Les jeux en ligne, du plaisir à l'addiction. Campus. Recuperado de http://www.unige.ch/communication/Campus/campus103/campus10 3.pdf

Ng, B. D. y Wiemer-Hastings, P. (2005). Addiction to the internet and online gaming. Cyberpsychology \& Behavior, 8, 110-113.

O'Neill, R. E., Horner, R. H., Albin, R. W., Storey, K., y Sprague, J. R. (1990). Functional Analysis of Problem Behavior: A Practical Assessment Guide. Sycamore IL: Sycamore Publishing Company

Orzack, M. H., Voluse, A. C., Wolf, W. y Hennen, D. (2006). An ongoing study of group treatment for men involved in problematic Internetenabled sexual behavior. Cyberpsychology \& Behavior, 9, 348-360.

Peng, W. y Liu, M. (2010). Online gaming dependency: A preliminary study in China. Cyberpsychology, Behavior and Social Networking, 13, 329-333.

Petry, N. M. (2006). Should the scope of addictive behaviors be broadened to include pathological gambling? Addiction, 101,152-160.

Phillips, C. A., Rolls, S., Rouse, A. ,y Griffiths, M. (1995). Home video game playing in schoolchildren: A study of incidence and patterns of play. Journal of Adolescence, 18, 687-691.

Potenza, M. N., Leung, H. C., Blumberg, H. P., Peterson, B. S., Fulbright, R. K., Lacadie, C. M., Skudlarski, P., y Gore, J.C. (2003). An fMRI Stroop task study of ventromedial prefrontal cortical function in pathological gamblers. American Journal of Psychiatry, 160, 1990-1994.

Rehbein, F., Psych, G., Kleimann, M., Mediasci, G., y Mossle, T. (2010). Prevalence and risk factors of video game dependency in adolescence: results of a German nationwide survey. CyberPsychology, Behavior and Social Networking, 13, 269-277.

Tejeiro, R. A. y Bersabé, R. M. (2002). Measuring problem video game playing in adolescents. Addiction, 97, 1601-1606

Thomas, N. J. y Martin, F. H. (2010). Video-arcade game, computer game and Internet activities of Australian students: participation habits and prevalence of addiction. Australian Journal of Psychology, 62, 59-66.

Van Rooij, A. J. (2011). Online Video Game Addiction. Exploring a new phenomenon (Tesis Doctoral). Erasmus University Rotterdam, The Netherlands. Recuperado de http://repub.eur.nl/res/pub/23381/.

Van Rooij, A., Schoenmakers, T., Vermulst, A., Van der Eijden, R., y Van de Mheen, D. (2010). Online video game addiction: Identification of addicted adolescent gamers. Addiction, 106, 205-212

Virués-Ortega, J. y Moreno-Rodríguez, R. (2008). Guidelines for clinical case reports in behavioral clinical psychology. International Journal of Clinical and Health Psychology, 8, 765-777

Wan, C. y Chiou, B. (2006b). Why are adolescents addicted to online gaming? An interview study in Taiwan. Cyberpsychology \& Behavior, 9, 762766. 
Wan, C. y Chiou, W. (2006a). Psychological motives and online games addiction: A test of flow theory and humanistic needs theory for Taiwanese adolescents. Cyberpsychology \& Behavior, 9, 317-324.

Wood, R. T. A. (2007). Problems with the concept of video game "addiction". Some case studies examples. International Journal of Mental Health and Addiction, 6, 169-178

Yee, N. (2006a). The demographics, motivations and derived experiences of users of massively-multiuser online graphical environments. Teleoperators and Virtual Environments, 15, 309-329.

Yee, N. (2006b). The psychology of MMORPGs: emotional investment, motivations, relationship formation, and problematic usage. En R. Schroeder y A. Axelsson (Eds.), Avatars at work and play: Collaboration and interaction in shared virtual environments (pp. 187-207). London: Springer.
Young, K. (1998). Internet addiction: The emergence of a new clinical disorder. CyberPsychology \& Behavior, 1, 237-244

Young, K. (2007). Cogntive-behavioral therapy with internet addicts: Treatment outcomes and implications. Cyberpsychology \& Behavior, 10, 671679.

Young, K. (2009). Understanding online gaming addiction and treatment issues for adolescents. American Journal of Family Therapy, 37, 355-372

Young, K.S. (2011). CBT-IA: The first treatment model for Internet Addiction. Journal of Cognitive Psychotherapy: An International Quarterly, 25, 304312

(Artículo recibido: 13-04-2012; revisado: 19-11-2012; aceptado: 19-11-2012)

\section{Anexo I. Evaluación del programa de tratamiento mediante preguntas abiertas}

A continuación se transcriben algunas de las preguntas realizadas por la terapeuta y las contestaciones de P. a cada una de ellas:

T: ¿Qué te ha parecido el tratamiento que has recibido?

P: "Bueno".

T: ¿Qué dificultades encontraste al inicio del tratamiento?

P: "Adaptarme a las normas que la terapeuta me impuso, como los horarios".

T: ¿Has notado cambios en tu estado de ánimo o en la forma en que te sientes?

P: "Si, estoy mejor".

$\mathrm{T}:$ ¿Has notado cambios en tu forma de pensar?

P: "Sí, puedo relativizar más algunas cosas".

T: ¿Has notado algún cambio en tu relación con los demás?

P: “No sé, supongo...".

T: ¿Qué habilidades crees que has aprendido?

P: "Visión un poco más objetiva del problema que be tenido y aplicarla en la vida real, y más autocontrol, sobre todo he ganado experiencia en este caso".

T: ¿Qué cambios has notado en la forma en que usas el ordenador, los videojuegos, Internet, las redes sociales...?

P: "Grandes cambios, de muchísimo que lo usaba ha bajado considerablemente el uso, sobre todo del juego".

T: ¿Qué crees que es lo más importante que has aprendido en terapia?

P. "Que un juego, o cualquier adicción se vuelve un problema grave cuando interfiere en la vida real de la persona llegándola a perjudicar seriamente, ya sea a nivel de estudios, familiar, etc.".

T: ¿Harías algún cambio sobre el tratamiento?

P: "No creo, está bien...tampoco se me ocurre nada". 